\title{
Anatomical variation of superior petrosal vein and its management during surgery for cerebellopontine angle meningiomas
}

\author{
Takao Watanabe • Takahiro Igarashi • Takao Fukushima • \\ Atsuo Yoshino • Yoichi Katayama
}

Received: 9 May 2013 / Accepted: 2 August 2013 / Published online: 30 August 2013

(C) The Author(s) 2013. This article is published with open access at Springerlink.com

\begin{abstract}
No systematic study is yet available that focuses on the surgical anatomy of the superior petrosal vein and its significance during surgery for cerebellopontine angle meningiomas. The aim of the present study was to examine the variation of the superior petrosal vein via the retrosigmoid suboccipital approach in relation to the tumor attachment of cerebellopontine angle meningiomas as well as postoperative complications related to venous occlusion. Forty-three patients with cerebellopontine angle meningiomas were analyzed retrospectively. Based on the operative findings, the tumors were classified into four subtypes: the petroclival type, tentorial type, anterior petrous type, and posterior petrous type. According to a previous anatomical report, the superior petrosal veins were divided into three groups: Type I which emptied into the superior petrosal sinus above and lateral to the internal acoustic meatus, Type II which emptied between the lateral limit of the trigeminal nerve at Meckel's cave and the medial limit of the facial nerve at the internal acoustic meatus, and Type III which emptied into the superior petrosal sinus above and medial to Meckel's cave. In both the petroclival and anterior petrous types, the most common vein was Type III which is the ideal vein for a retrosigmoid approach. In contrast, the Type II vein which is at high risk of being sacrificed during a suprameatal approach procedure was most frequent in posterior petrous type, in which the superior petrosal vein was not largely an obstacle. Intraoperative sacrificing of veins was associated with a significantly higher rate of venous-related phenomena, while venous complications occurred even in cases where the superior petrosal vein
\end{abstract}

T. Watanabe $(\bowtie) \cdot$ T. Igarashi $\cdot$ T. Fukushima $\cdot$ A. Yoshino

Y. Katayama

Department of Neurological Surgery, Nihon University School of

Medicine, 30-1 Oyaguchi-kamimachi, Itabashi-ku,

Tokyo 173-8610, Japan

e-mail: takao@med.nihon-u.ac.jp was absent or compressed by the tumor. The variation in the superior petrosal vein appeared to differ among the tumor attachment subtypes, which could permit a satisfactory surgical exposure without dividing the superior petrosal vein. In cases where the superior petrosal vein was previously occluded, other bridging veins could correspond with implications for the crucial venous drainage system, and should thus be identified and protected whenever possible.

Keyword Attachment . Classification · Cerebellopontine angle $\cdot$ Meningioma $\cdot$ Petrosal vein $\cdot$ Venous complication

\section{Introduction}

The petrosal vein complex is at risk of being sacrificed during the microsurgical removal of extra-axial cerebellopontine angle tumors, particularly meningiomas involving the petrous apex, because of its close vicinity or adherence to the tumor. Coagulation and dissection of the superior petrosal vein do not frequently cause adverse sequelae, but a recent study on a substantial number of patients with petrous apex meningiomas has revealed minor venous congestion phenomena in $23 \%$ and major complications in almost $7 \%$ after intraoperative obliteration of the superior petrosal vein [2], emphasizing the view that preservation of the petrosal venous complex is still the best way of reducing the likelihood of venous complications. Nevertheless, the superior petrosal vein entering that part of the superior petrosal sinus above the suprameatal tubercle often has to be sacrificed when the suprameatal extension of the retrosigmoid approach is employed to expose Meckel's cave and the posterior part of the middle fossa $[8,9]$.

In a recent study on anatomical dissections using cadaveric specimens, Tanriover et al. [9] classified the drainage patterns of the superior petrosal venous complex on the basis of the 
relationship between their site of entry into the superior petrosal sinus, Meckel's cave, and the internal acoustic meatus. According to their observations, approximately $90 \%$ of cases during the retrosigmoid suprameatal approach procedure required the division of the superior petrosal veins in order to achieve a satisfactory surgical exposure along Meckel's cave and the middle fossa dura. This proposed classification system for the superior petrosal venous complex could aid in planning the approach directed along the petrous apex and reducing the likelihood of venous complications, although their findings obtained from cadaveric dissections cannot be universally adopted for the actual surgical exposures in petrous apex meningiomas, because in the majority of their tumors the superior petrosal vein is likely to have been markedly displaced and/or compressed. The literature unfortunately does not yet provide any quantitative data concerning the frequency or type of anatomical variations of the superior petrosal vein during surgery for cerebellopontine angle meningiomas.

The aim of the present study was to examine the surgical anatomy of the superior petrosal vein via the retrosigmoid suboccipital approach in relation to the tumor attachment of cerebellopontine angle meningiomas. We retrospectively analyzed 43 cases of cerebellopontine angle meningiomas treated surgically at our institution. The postoperative complications related to venous occlusion were also assessed.

\section{Clinical materials and methods}

\section{Patient population}

A total of 43 patients with meningiomas at the cerebellopontine angle who were treated surgically at our institution between April 1998 and March 2012 were included in this study. Although two neurosurgeons (T.W. and Y.K.) were involved, all of the patients underwent operations by or under the supervision of one neurosurgeon (Y.K.). Tumors with obvious attachment to the lower clivus, jugular foramen, or foramen magnum, and tentorial meningiomas unrelated to the cerebellopontine angle were excluded from the present study. Cases with previous surgical treatment were also excluded because considerable alterations to the venous drainage would be present due to the first operation. Careful retrospective reviews of the patients' clinical records and radiological images were carried out. In addition, surgical video recordings of all cases were reviewed retrospectively and evaluated in order to classify the superior petrosal venous complex and form of tumor attachment.

All patients underwent a standard suboccipital retrosigmoid approach in the lateral oblique position. In the majority of cases, suprameatal extension (suprameatal approach) and/or tentorial incision (transtentorial approach) was added to this approach employing techniques described elsewhere $[7,8,10]$.
Tumor attachment, superior petrosal vein, and venous complications

Based on the operative records and surgical video recordings, the attachment of the tumor was classified into four subtypes: petroclival, tentorial, anterior petrous, and posterior petrous, as reported previously $[1,3]$. The petroclival-type category tumors are attached medially to the trigeminal nerve. The trigeminal nerve in this type is shifted laterally. The tentorial type originates from the medial tentorium. The trigeminal nerve is shifted inferomedially. The anterior petrous type is where the tumor is attached anterior to the internal acoustic meatus. The trigeminal nerve is shifted superomedially. The posterior petrous type is where the tumor is attached posterior to the internal acoustic meatus.

According to the classification of Tanriover et al. [9], the superior petrosal veins were divided into three groups (Fig. 1). In Type I, the stem of the superior petrosal venous complex emptied into the superior petrosal sinus above or lateral to the boundaries of the internal acoustic meatus, at a point lateral to the medial limit of the facial nerve at its point of entry into the internal acoustic meatus. In Type II, the superior petrosal venous complex emptied into an area between the lateral limit of the trigeminal nerve at its point of entry at Meckel's cave and the medial limit of the facial nerve at its point of entry at the internal acoustic meatus. In Type III, the superior petrosal venous complex emptied into the superior petrosal sinus above or medial to the boundaries of Meckel's cave, at a point

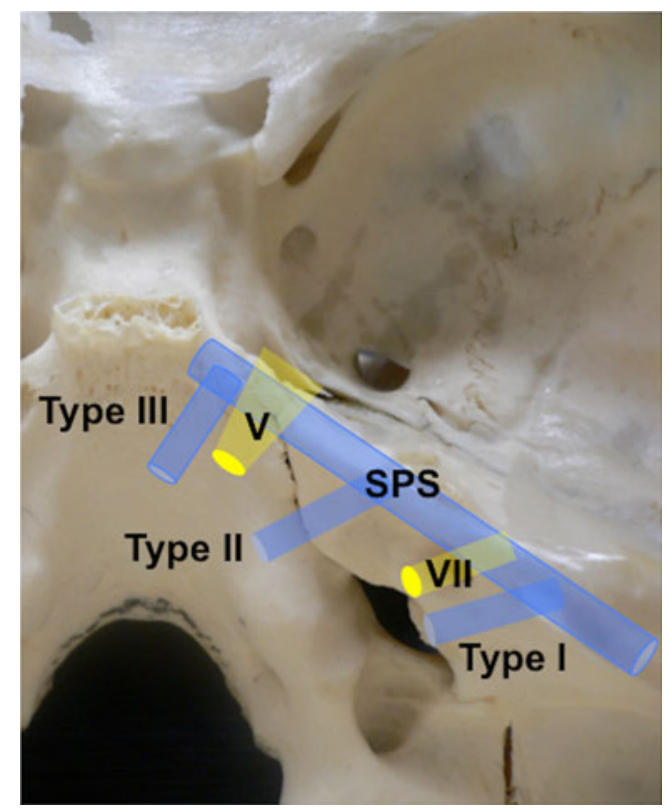

Fig. 1 Three types of drainage of the superior petrosal vein. Type I empties into the superior petrosal sinus above and lateral to the facial nerve. Type II empties between the lateral limit of the trigeminal nerve and the medial limit of the facial nerve. Type III empties into the superior petrosal sinus above and medial to the trigeminal nerve; SPS superior petrosal sinus 
medial to the lateral limit of the trigeminal nerve at its site of entry into Meckel's cave. The numbers of the veins, presence of venous compression by the tumor, and surgical preservation of the veins versus sacrifice were also evaluated from the intraoperative findings.

The postoperative outcome analysis considered all complications related to venous occlusion, such as cerebellar edema, venous infarction, and hemorrhagic infarction. According to the description of Koerbel et al. [2], cerebellar edema was defined as involving compression of the fourth ventricle and quadrigeminal cistern with ventricular enlargement.

\section{Statistical analysis}

The relationships between the various parameters were analyzed statistically using the chi-square test, Fisher's exact test, or Mann-Whitney $U$-test as appropriate. Multivariate regression analysis was not performed because of the small number of patients included. The level of significance level was chosen at $P<0.05$, and all tests were two-sided.

\section{Results}

The patients included 20 men and 23 women. Their mean age was 48 years and ranged from 15 to 75 years. None of the patients had undergone any previous surgical procedure. Pathological tumor grade information was available for all 43 patients: 42 cases were of Grade I, and one was of Grade II on the World Health Organization (WHO) classification. The standard retrosigmoid craniotomy was extended via a suprameatal approach in 6 , a transtentorial approach in 7 , and both suprameatal and transtentorial approaches in 20 of the 43 cases. There were no cases of operative death in the present surgical series of cerebellopontine angle meningiomas. Table 1 summarizes the characteristics of the 43 cases composing the series.

Tumor attachment and superior petrosal vein

According to the attachment classification, the petroclival, tentorial, anterior petrous, and posterior petrous subtypes included $22(51 \%), 5(12 \%), 7$ (16\%), and 9 (21\%) tumors, respectively (Table 1). Among the 43 cases examined in our study, the superior petrosal vein was not identified in six cases during the surgery. All of these 6 tumors were greater than $31 \mathrm{~mm}$ in diameter (range, 32 to $52 \mathrm{~mm}$; median, $46 \mathrm{~mm}$ ). In the remaining 37 cases, a single petrosal vein was identified in $31(84 \%)$ cases, whereas two or more veins were observed in $6(16 \%)$ patients. These 37 patients were successfully classified into one of the three petrosal vein types: they included 3 patients ( $8 \%$ ) with Type I, 15 patients (41\%) with Type II, and 19 patients (51\%) with Type III (Fig. 2). Among the 37
Table 1 Summary of 43 cases of cerebellopontine angle meningiomas

\begin{tabular}{|c|c|c|c|c|}
\hline & Petroclival & Tentorial & $\begin{array}{l}\text { Anterior } \\
\text { petrous }\end{array}$ & $\begin{array}{l}\text { Posterior } \\
\text { petrous }\end{array}$ \\
\hline No. of patients & 22 & 5 & 7 & 9 \\
\hline \multicolumn{5}{|l|}{ Age (years) } \\
\hline Range & $15-70$ & $39-75$ & $32-67$ & $32-66$ \\
\hline Median & 53 & 57 & 58 & 56 \\
\hline \multicolumn{5}{|l|}{ Gender } \\
\hline Male & 10 & 2 & 3 & 4 \\
\hline Female & 12 & 3 & 4 & 5 \\
\hline \multicolumn{5}{|l|}{ Size (mm) } \\
\hline Range & $22-48$ & $23-47$ & $22-52$ & $23-46$ \\
\hline Median & 28 & 32 & 28 & 28 \\
\hline \multicolumn{5}{|l|}{ SPV type } \\
\hline Type I & 1 & 1 & 0 & 1 \\
\hline Type II & 6 & 2 & 2 & 2 \\
\hline Type III & 12 & 1 & 4 & 5 \\
\hline Not identified & 3 & 1 & 1 & 1 \\
\hline \multicolumn{5}{|l|}{ Number of SPVs } \\
\hline Single & 16 & 3 & 5 & 7 \\
\hline Two or more & 3 & 1 & 1 & 1 \\
\hline \multicolumn{5}{|c|}{ SPV compression to the tumor } \\
\hline Yes & 5 & 3 & 2 & 2 \\
\hline No & 14 & 1 & 4 & 6 \\
\hline \multicolumn{5}{|c|}{ Preservation of SPV } \\
\hline Yes & 11 & 1 & 5 & 7 \\
\hline No & 8 & 3 & 1 & 1 \\
\hline \multicolumn{5}{|l|}{ Approach } \\
\hline Simple & 0 & 0 & 1 & 9 \\
\hline SM & 3 & 0 & 3 & 0 \\
\hline TT & 3 & 4 & 0 & 0 \\
\hline $\mathrm{SM}+\mathrm{TT}$ & 16 & 1 & 3 & 0 \\
\hline
\end{tabular}

SPV superior petrosal vein; SM suprameatal approach; TT transtentorial approach.

patients with identified petrosal veins, compression by the tumor was noted in $12(34 \%)$ cases. Although no statistically significant difference was found ( $P=0.4034$ on the chi-square test), the proportion of Type III was higher for the petroclival and anterior petrous types (63\% and $67 \%$, respectively). In contrast, Type II was most frequent in tumors with a posterior petrous type attachment (63\%). Neither age, sex, tumor size, number of veins, venous compression, nor extension of approach was significantly correlated with the attachment classification ( $P>0.5$ for each comparison; data not shown).

Vein preservation

For the petroclival tumors, the superior petrosal vein complex could be preserved in 11 (58\%) out of 19 patients with identified veins. In the majority of the Type III cases, the superior petrosal 

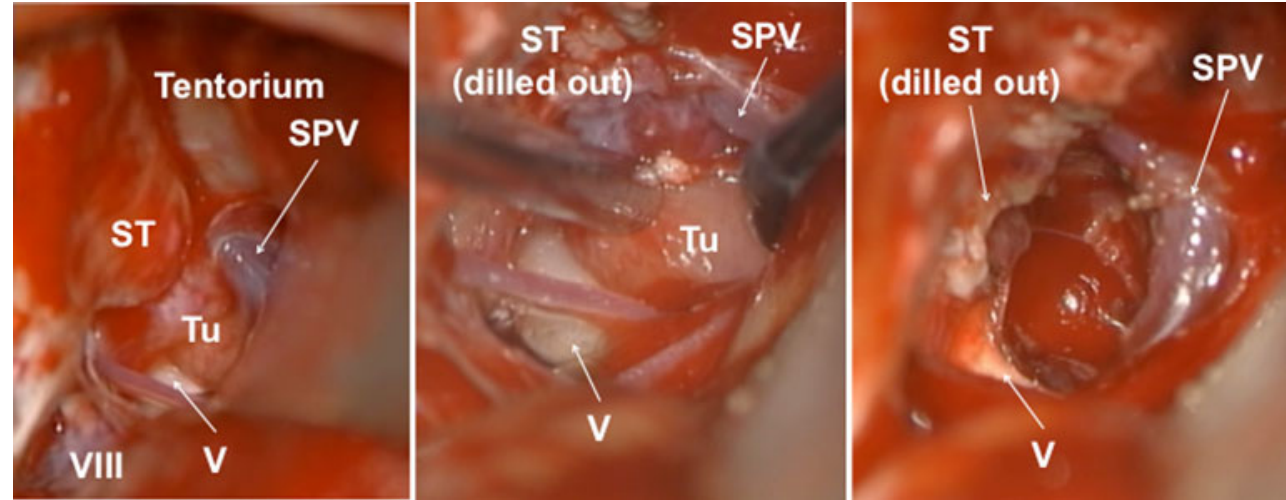

Fig. 2 Intraoperative views demonstrating removal of petroclival meningioma via the left lateral suboccipital approach. Left: The tumor has an attachment medially to the trigeminal nerve. The superior petrosal vein empties into the superior petrosal sinus medial to the trigeminal nerve, indicating Type III. Center: With drilling the suprameatal bulging of the

veins were not an obstacle for the suprameatal and/or transtentorial approach: the complete superior petrosal vein complex could be preserved in $10(83 \%)$ of the 12 cases. In contrast, for Types I and II, at least one petrosal vein needed to be obliterated in all but one case with Type II in order to gain sufficient working space along Meckel's cave and the tentorium.

For the tentorial tumors, the superior petrosal veins could not be preserved in all except one case with Type I (preservation rate $25 \%$ ).

For the anterior petrosal tumors, complete preservation of the superior petrosal vein complex was achieved in all patients except one having a Type II vein (preservation rate, $83 \%$ ).

For the posterior petrosal type, we employed a simple retrosigmoid approach without any procedure for drilling the suprameatal bone or incising the tentorium, and gross total resection was achieved in all cases. The superior petrosal vein complex could be completely preserved in all patients except for the one having a Type I vein (preservation rate $88 \%$ ).

Overall, in $13(35 \%)$ out of the 37 cases with identified veins, at least one petrosal vein was sacrificed in order to increase the surgical exposure during removal of the tumor. Neither tumor attachment subtype nor approach extension revealed a correlation with preservation of the superior petrosal vein complex. Surgical preservation of the vein versus sacrifice was significantly associated with tumor size, venous compression by the tumor, and petrosal vein subtype (Table 2).

\section{Venous complications}

Postoperative venous-related complications occurred In 5 (12\%) of the 43 cases (Table 3 ). Three of these 5 cases had cerebellar edema with ventricular enlargement. This complication resolved itself within a few days following conservative therapy, although intensive care management with placement of an external ventricular drainage was required in two cases. petrous bone, the tumor is dissected between the superior petrosal vein and trigeminal nerve. Right: After tumor resection, preservation of the superior petrosal vein is accomplished. SPV superior petrosal vein; ST suprameatal tubercle

Table 2 Analysis of the association between various factors and preservation of the superior petrosal vein versus sacrifice in 37 patients with identified veins

\begin{tabular}{|c|c|c|c|}
\hline & $\begin{array}{l}\text { Preserved } \\
\text { (24 patients) }\end{array}$ & $\begin{array}{l}\text { Sacrificed } \\
\text { (13 patients) }\end{array}$ & $P$ value \\
\hline \multicolumn{4}{|l|}{ Age (years) } \\
\hline Range & $27-64$ & $15-75$ & \multirow[t]{2}{*}{0.2028} \\
\hline Median & 51.5 & 59 & \\
\hline \multicolumn{4}{|l|}{ Gender } \\
\hline Male & 11 & 6 & \multirow[t]{2}{*}{0.9851} \\
\hline Female & 13 & 7 & \\
\hline \multicolumn{4}{|l|}{ Size $(\mathrm{mm})$} \\
\hline Range & $22-38$ & $27-48$ & \multirow[t]{2}{*}{0.0021} \\
\hline Median & 26.5 & 34 & \\
\hline \multicolumn{4}{|l|}{ Tumor type } \\
\hline Petroclival & 11 & 8 & \multirow[t]{4}{*}{0.1170} \\
\hline Tentorial & 1 & 3 & \\
\hline Anterior petrous & 5 & 1 & \\
\hline Posterior petrous & 7 & 1 & \\
\hline \multicolumn{4}{|l|}{ Vein type } \\
\hline Type I & 1 & 2 & \multirow[t]{3}{*}{0.0367} \\
\hline Type II & 7 & 8 & \\
\hline Type III & 16 & 3 & \\
\hline \multicolumn{4}{|l|}{ Venous compression } \\
\hline Yes & 3 & 9 & \multirow[t]{2}{*}{0.0008} \\
\hline No & 21 & 4 & \\
\hline \multicolumn{4}{|l|}{ Approach } \\
\hline Simple & 8 & 1 & \multirow[t]{4}{*}{0.2054} \\
\hline SM & 6 & 2 & \\
\hline TT & 2 & 2 & \\
\hline $\mathrm{SM}+\mathrm{TT}$ & 8 & 8 & \\
\hline
\end{tabular}

SM suprameatal approach; TT transtentorial approach. 
Table 3 Summary of cases presenting with postoperative venous complications

\begin{tabular}{llllllllll}
\hline Case & $\begin{array}{l}\text { Age } \\
\text { (years })\end{array}$ & Gender & $\begin{array}{l}\text { Tumor size } \\
(\mathrm{mm})\end{array}$ & $\begin{array}{l}\text { Attachment } \\
\text { type }\end{array}$ & SPV type & $\begin{array}{l}\text { Vein } \\
\text { compression }\end{array}$ & $\begin{array}{l}\text { Preservation } \\
\text { of SPV }\end{array}$ & Approach & Complications \\
\hline 1 & 64 & F & 48 & Petroclival & Type II & No & No & SM+TT & Venous infarction \\
2 & 61 & F & 27 & Petroclival & Type II & No & No & SM+TT & Transient edema \\
3 & 34 & M & 42 & Petroclival & Type II & No & No & SM+TT & Transient edema \\
4 & 69 & F & 46 & Petroclival & Not identified & & & SM+TT & Transient edema \\
5 & 75 & F & 32 & Tentorial & Type II & Yes & No & TT & Hemorrhagic infarction \\
\hline
\end{tabular}

M male, F female, SPV superior petrosal vein, SM suprameatal approach, TT transtentorial approach.

In one patient (case 5), postoperative cerebellar hemorrhagic infarction presented a life-threatening condition and had to be operated on for posterior fossa decompression with removal of the necrotic cerebellar tissue (Fig. 3). In this case, a prominent superior hemispheric vein coursed anterolaterally to drain into the tentorial sinus that joined the junction of the superior petrosal and transverse sinuses, and was divided through a lateral supracerebellar infratentorial avenue (Fig. 4). Another patient developed a cerebellar venous infarction, leading to prolonged intensive care treatment with a delayed postoperative recovery.

Among the six cases where no petrosal vein could be identified, one experienced transient cerebellar edema postoperatively. No postoperative complications related to venous congestion were encountered in any of the 24 patients in whom the petrosal vein complex was preserved. In contrast, venous complications occurred in 4 (31\%) of the 13 cases where at least one petrosal vein was occluded. This difference in incidence of complications between the sacrificed-vein group and preserved-vein group was significant $(P=0.0108$ on Fisher's exact test). The petrosal vein type also influenced the occurrence of postoperative complications: venous complications occurred in 4 (27\%) of the 15 cases with Type II, but such complications were not observed in any of the three cases with Type I and 19 cases with Type III ( $P=0.0373$ on the chi-square test). Other factors including age, gender, tumor size, tumor attachment, and petrosal vein compression by the tumor did not have a significant impact on the incidence of postoperative venous complications $(P>0.1$ for each comparison; data not shown).

\section{Discussion}

Although the microsurgical anatomy of the superior petrosal vein has been extensively examined based on cadaveric dissections and a classification exists in previous anatomical reports $[5,9]$, a detailed description of the superior petrosal vein in relation to the tumor attachment of posterior fossa meningiomas has not yet been given. The present study was therefore focused mainly on the surgical anatomy of the superior petrosal vein together with its significance during surgery for cerebellopontine angle meningiomas.

The drainage pattern of the superior petrosal vein appears to vary among the different attachment subtypes. In both the petroclival and anterior petrous types, the most frequent venous pattern was Type III (63\% and $67 \%$, respectively) which would represent the ideal vein for a retrosigmoid suprameatal approach. In the classification proposed by Tanriover et al. [9] who used cadaveric specimens, however, Type III was the least ordinary pattern $(9 \%)$, and their most common vein was Type II (72\%) which could limit the amount of working space available during the retrosigmoid approach procedure. In contrast to the petroclival and anterior petrous types, Type II was most frequently encountered in the posterior petrous type ( $63 \%$ of cases). In views of the fact that all of our cases where no petrosal vein could be identified had a relatively huge size of tumor, identified petrosal veins could be in broad hemodynamic balance with other venous structures being compressed or displaced by the tumor. Although the limitations of the present relatively small sample size preclude any firm conclusions, differences in venous pattern might reflect the distortion of the normal anatomic venous structures and development of venous collateral circulations in each tumor subtype, rather than merely individual variations of the normal anatomy of the superior petrosal vein.

In general, preservation of the superior petrosal vein is not always achievable in neurosurgical procedures, especially when the standard suboccipital approach is modified with suprameatal and/or supratentorial extension [8,9]. In our series, extension of approach did not have a significant impact on the risk of vein sacrifice. This may be possibly due to the fact that the superior petrosal vein did not represent an obstacle for the suboccipital retrosigmoid approach in most cases except for the tentorial type. The superior petrosal vein complex could be preserved in $88 \%, 83 \%$, and $58 \%$ of our patients with the posterior petrous type, anterior petrous type, and petroclival type, respectively, while complete preservation of the superior petrosal vein complex was achieved in only $25 \%$ of patients with the tentorial type. Such high frequency of vein preservation may be attributable to venous variation among the different attachment subtypes of cerebellopontine angle meningiomas. 

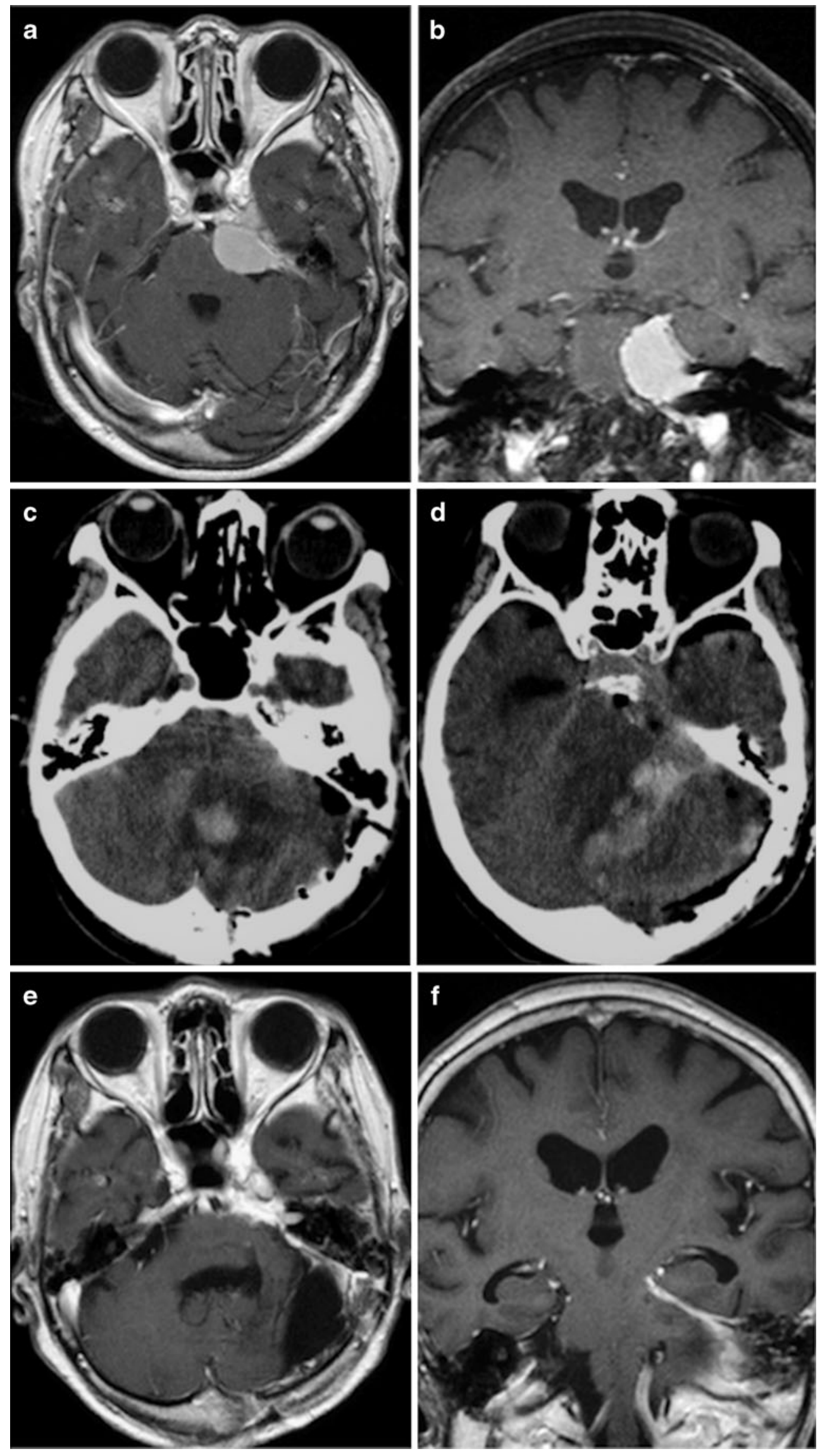
4 Fig. 3 Representative patient (case 5) with life-threatening venous complications. Preoperative contrast-enhanced axial (a) and coronal (b) magnetic resonance (MR) images revealed a homogeneous mass at the left cerebellopontine angle with attachment to the tentorium. Postoperative computed tomography scans obtained at one day after the first operation (c and d) demonstrated intracerebellar hemorrhage and swelling. Postoperative contrast-enhanced axial (e) and coronal (f) MR images obtained at 3 months after the operation showed total tumor resection and atrophic cerebellum

In the present study, intraoperative obliteration of the superior petrosal vein was associated with a significantly higher rate of postoperative venous-related phenomena. Venous complications occurred in up to $31 \%$ of cases in which the superior petrosal vein was sacrificed. This frequency was similar to that in the previous report [2], emphasizing the importance of preserving the superior petrosal vein. However, its sacrifice does not necessarily give rise to serious problems in most cases [2, 4], and a method for detecting the risk of developing postoperative deficits after petrosal vein obliteration has not yet been established. The wide anatomical variability, e.g., in vein number, size, and anatomic connections, is probably largely responsible for the inconsistent occurrence of postoperative complications.

In the present series, postoperative venous-related complications occurred even in the case where the superior petrosal vein was absent. In such a situation, there might be other unnamed bridging veins running from the cerebellum to the dura that should be identified and protected whenever possible, although our retrospective reviews of the surgical video recordings failed to identify any responsible vein. As exemplified by our illustrative case (case 5), the superior petrosal vein appeared already to be significantly occluded by the tumor itself so that sacrificing the last remaining vein could give rise to severe morbidity. In this case, a prominent superior hemispheric vein ran laterally to drain into the junction of the superior petrosal and transverse sinuses, and was inevitably sectioned through a lateral supracerebellar infratentorial avenue, which could ultimately lead to significant cerebellar impairment. According to previous anatomical reports $[5,6]$, superior hemispheric veins are usually situated in the medial portion of the tentorium, and may thus not represent an obstacle in the lateral suboccipital approach. In such an unfavorable situation, the retrosigmoid suboccipital approach should be abandoned, and an alternative approach, i.e., the anterior transpetrosal approach, would be indicated to preserve this crucial venous drainage.

In conclusion, the variation in the superior petrosal vein appears to differ among the attachment subtypes of cerebellopontine angle meningiomas. In the majority of cases except for the tentorial subtype, an adequate surgical exposure for the suboccipital retrosigmoid approach was achieved without dividing the superior petrosal vein. Intraoperative sacrificing of the superior petrosal vein was significantly associated with the occurrence of venous-related phenomena, while venous complications occurred even in cases where the superior petrosal vein was absent or already occluded by the tumor itself. In such cases, other bridging veins could play a critical role in the venous drainage system of the posterior fossa. In patients who might be considered to be at risk, an alternative approach may enhance the possibility of preserving the crucial venous drainage and thus reducing the morbidity.

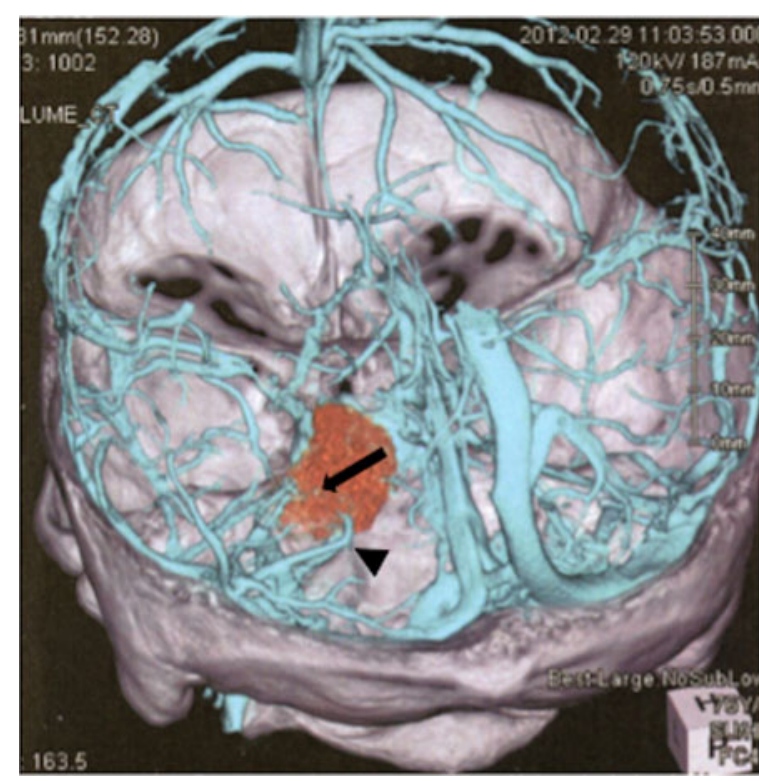

Fig. 4 Posterosuperior views of the venous phase of three-dimensional computed tomography angiograms obtained in the patient illustrated in Fig. 2. The superior petrosal vein was entirely encased by the tumor

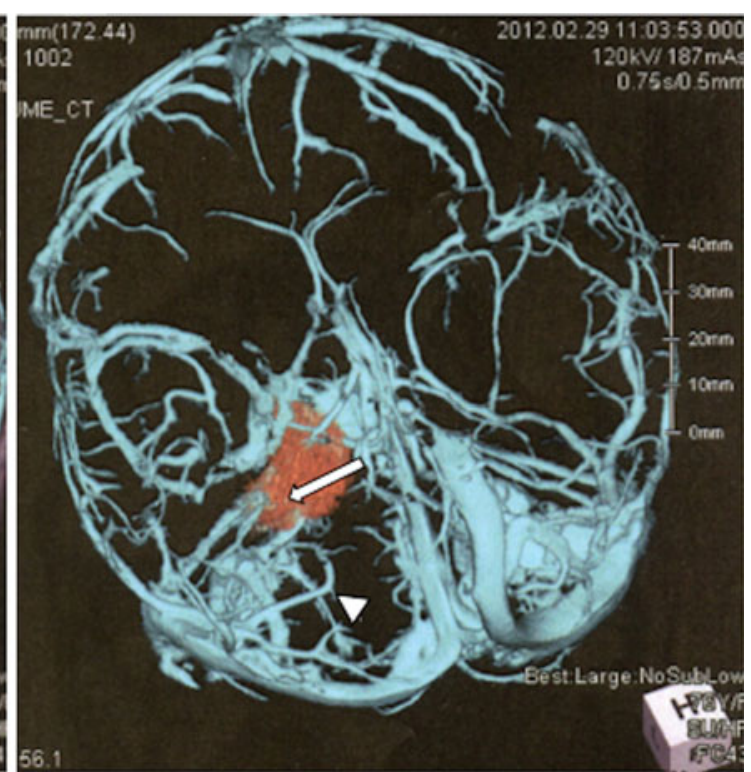

(arrow). The superior hemispheric vein was formed by a bridging vein running anterolaterally to drain into the tentorial sinus that jointed the junction of the superior petrosal and transverse sinuses (arrowhead) 
Conflicts of interest The authors report no conflict of interest concerning the materials or methods used in this study or the findings specified in this paper.

Open Access This article is distributed under the terms of the Creative Commons Attribution License which permits any use, distribution, and reproduction in any medium, provided the original author(s) and the source are credited.

\section{References}

1. Ichimura S, Kawase T, Onozuka S, Yoshida K, Ohira T (2008) Four subtypes of petroclival meningiomas: differences in symptoms and operative findings using the anterior transpetrosal approach. Acta Neurochir (Wein) 150:637-645

2. Koerbel A, Gharabaghi A, Safavi-Abbasi S, Samii A, Ebner FH, Samii M, Tatagiba M (2009) Venous complications following petrosal vein sectioning in surgery of petrous apex meningiomas. Eur J Surg Oncol 35:773-779

3. Kunii N, Ota T, Kin T, Kameda K, Morita A, Kawahara N, Saito N (2011) Angiographic classification of tumor attachment of meningiomas at the cerebellopontine angle. World Neurosurg 75:114-121

4. Masuoka J, Matsushima T, Hikita T, Inoue E (2009) Cerebellar swelling after sacrifice of the superior petrosal vein during microvascular decompression for trigeminal neuralgia. J Clin Neurosci 16:1342-1344

5. Matsushima T, Rhoton AL Jr, de Oliveira E, Peace D (1983) Microsurgical anatomy of the veins of the posterior fossa. J Neurosurg 59:63-105

6. Matsushima T, Suzuki SO, Fukai M, Rhoton AL Jr, de Oliveira E, Ono M (1989) Microsurgical anatomy of the tentorial sinuses. J Neurosurg 71:923-928
7. Samii M, Tatagiba M, Carvalho GA (2000) Retrosigmoid intradural suprameatal approach to Meckel's cave and the middle fossa: surgical technique and outcome. J Neurosurg 92:235-241

8. Seoane E, Rhoton AL Jr (1999) Suprameatal extension of the retrosigmoid approach: microsurgical anatomy. Neurosurgery 44: $553-560$

9. Tanriover N, Abe H, Rhoton AL Jr, Kawashima M, Sanus GZ, Akar Z (2007) Microsurgical anatomy of the superior petrosal venous complex: new classifications and implications for subtemporal transtentorial and retrosigmoid suprameatal approaches. J Neurosurg 106:1041-1050

10. Watanabe T, Katayama Y, Fukushima T, Kawamata T (2011) Lateral supracerebellar transtentorial approach for petroclival meningiomas: operative technique and outcome. J Neurosurg 115:49-54

\section{Comment}

In this paper Watanabe et al. performed a clinic-anatomical study on the variants of the superior petrosal vein examining the neuroimaging and the surgical video recording of a series of patients treated at their Institution for cerebellopontine angle meningiomas.

They retrospectively reviewed 43 cases investigating the specific association found between the different venous patterns and the lesions.

Moreover, they also validated the evidence that intraoperative sacrificing of superior petrosal veins was associated with a significantly higher rate of venous-related phenomena as recently demonstrated by other authors in literature.

This paper is interesting because it emphasizes once again the importance of sparing the venous drainage during surgery in this area.

Domenico d'Avella

Padova, Italy 